ARTICLE

\title{
Topological charge-entropy scaling in kagome Chern magnet $\mathrm{TbMn}_{6} \mathrm{Sn}_{6}$
}

\author{
Xitong Xu1,2, Jia-Xin Yin ${ }^{3}$, Wenlong Ma (i) ${ }^{2}$, Hung-Ju Tien (10) 4,5, Xiao-Bin Qiang (1) 6, P. V. Sreenivasa Reddy (1D ${ }^{4}$, \\ Huibin Zhou ${ }^{2}$, Jie Shen ${ }^{1,7}$, Hai-Zhou Lu6 ${ }^{6}$, Tay-Rong Chang4,5,8, Zhe Qu (D) 1,7,9凶 \& Shuang Jia (D) 2,10,11凶
}

In ordinary materials, electrons conduct both electricity and heat, where their charge-entropy relations observe the Mott formula and the Wiedemann-Franz law. In topological quantum materials, the transverse motion of relativistic electrons can be strongly affected by the quantum field arising around the topological fermions, where a simple model description of their charge-entropy relations remains elusive. Here we report the topological charge-entropy scaling in the kagome Chern magnet $\mathrm{TbMn}_{6} \mathrm{Sn}_{6}$, featuring pristine Mn kagome lattices with strong out-of-plane magnetization. Through both electric and thermoelectric transports, we observe quantum oscillations with a nontrivial Berry phase, a large Fermi velocity and twodimensionality, supporting the existence of Dirac fermions in the magnetic kagome lattice. This quantum magnet further exhibits large anomalous Hall, anomalous Nernst, and anomalous thermal Hall effects, all of which persist to above room temperature. Remarkably, we show that the charge-entropy scaling relations of these anomalous transverse transports can be ubiquitously described by the Berry curvature field effects in a Chern-gapped Dirac model. Our work points to a model kagome Chern magnet for the proof-of-principle elaboration of the topological charge-entropy scaling.

\footnotetext{
${ }^{1}$ Anhui Key Laboratory of Condensed Matter Physics at Extreme Conditions, High Magnetic Field Laboratory, Hefei Institutes of Physical Science, Chinese Academy of Sciences, Hefei, Anhui 230031, China. ${ }^{2}$ International Center for Quantum Materials, School of Physics, Peking University, Beijing 100871, China. ${ }^{3}$ Department of Physics, Princeton University, Princeton, New Jersey 08544, USA. ${ }^{4}$ Department of Physics, National Cheng Kung University, Tainan 701, Taiwan. ${ }^{5}$ Center for Quantum Frontiers of Research and Technology (QFort), Tainan 701, Taiwan. ${ }^{6}$ Department of Physics and Shenzhen Institute for Quantum Science and Engineering, Southern University of Science and Technology, Shenzhen 518055, China. ${ }^{7}$ Science Island Branch of Graduate School, University of Science and Technology of China, Hefei, Anhui 230026, China. ${ }^{8}$ Physics Division, National Center for Theoretical Sciences, Taipei 10617 , Taiwan. ${ }^{9}$ CAS Key Laboratory of Photovoltaic and Energy Conservation Materials, Hefei Institutes of Physical Science, Chinese Academy of Sciences, Hefei, Anhui 230031, China. ${ }^{10}$ Interdisciplinary Institute of Light-Element Quantum Materials and Research Center for Light-Element Advanced Materials, Peking University, Beijing 100871, China. ${ }^{11}$ CAS Center for Excellence in Topological Quantum Computation, University of Chinese Academy of Sciences, Beijing 100190, China. $\bigotimes_{\text {email: }}$ hequ@hmfl.ac.cn; gwljiashuang@pku.edu.cn
} 
E lucidating the quantum nature and transport behaviors of topological magnets is at the frontier of condensed matter physics, and provides indispensable information on the application of quantum material research ${ }^{1-5}$. The interplay between topology and magnetism can naturally occur in kagome lattice systems ${ }^{6,7}$. Owing to its special lattice geometry, the electronic structure of kagome lattice inherently features both Dirac cones and band singularities including flat bands and van Hove singularities. The former provides the source of topological nontrivial band structure, while the latter can drive magnetic instabilities. In this regard, several transition metal based kagome magnets are of current interest, which exhibit many-body interplays and Chern quantum phases ${ }^{8-18}$. Among these kagome magnets, $\mathrm{TbMn}_{6} \mathrm{Sn}_{6}$ stands out owing to its pristine kagome lattice (without other atoms in the kagome lattice plane) and strong outof-plane magnetization (persisting to above room temperature). Spatial and momentum resolved spectroscopies have uncovered the topological electronic structure that can be described by a simple Chern-gapped Dirac model ${ }^{16,19-21}$. The tight-binding model of kagome lattice considering the nearest neighbouring hopping hosts Dirac cones at the Brillouin zone corners. Magnetic exchange interaction splits the spin-up and spin-down Dirac cones, while the combination of out-of-plane magnetization and KaneMele type spin-orbit coupling further opens the Chern gap for the spin-polarized Dirac fermions. Though the spectroscopic and some preliminary electric transport data have been reported ${ }^{16,17}$, the quantum transport behavior of this kagome Chern magnet remains largely unveiled, which questions the key topological transport features of Chern-gapped Dirac fermions. Here we use the combination of electric, thermoelectric and thermal transport to characterize this quantum magnet and to discover its topological charge-entropy scaling.

\section{Results}

Parametrization of the Chern-gapped Dirac fermion. $\mathrm{TbMn}_{6} \mathrm{Sn}_{6}$ crystallizes in the space group of $\mathrm{P} 6 / \mathrm{mmm}$, featuring a pure $\mathrm{Mn}$-based kagome lattice. Below its Curie temperature $(\sim 420 \mathrm{~K})$, it possesses a ferrimagnetic state in which the magnetic moment of $\mathrm{Tb}$ is anti-parallel aligned with the ferromagnetic ordered Mn lattice due to the strong exchange coupling between $\mathrm{Tb}$ and $\mathrm{Mn}$ moments. The ferrimagnetic state changes its anisotropy from an easy-plane to an out-of-plane configuration when the temperature is below $313 \mathrm{~K}$. The out-of-plane ferromagnetic Mn kagome lattice, which is stable over a wide range in the phase diagram (Fig. 1a), is crucial to support the fully spinpolarized Dirac fermions with a large Chern gap ${ }^{16,17}$. Figure $1 b$ shows the quantum oscillations resolved in the thermoelectric and electric transport measurements at low temperatures. In the Seebeck signals, strong quantum oscillations with one dominant frequency $(\alpha, 96 \mathrm{~T})$ can be observed just above $5 \mathrm{~T}$, which is the smallest field among all known kagome magnets. The overall oscillatory patterns between thermopower and resistance show a $\pi / 2$ phase shift, indicating that the $\alpha$ orbit is from a hole-like band $^{22}$, consistent with spectroscopic observations ${ }^{16}$. The cyclotron mass of $\alpha$ orbit is found to be $0.14 m_{e}$ by fitting to the Lifshitz-Kosevich formula (See Method section). According to the Onsager relation ${ }^{23}$, this frequency corresponds to a Fermi vector $k_{F}$ of $0.05 \AA^{-1}$. The (Dirac cone) velocity $v_{D}=\hbar k_{F} / m^{*}$ is as large as $4.2 \times 10^{5} \mathrm{~m} / \mathrm{s}$, and the (Dirac cone) energy $E_{D} \simeq m^{*} \nu_{D}^{2}$ is estimated to be around $140 \mathrm{meV}$, in remarkable accordance with the hole branch of the Dirac dispersion observed in the previous spectroscopic experiments ${ }^{16}$. We further subtract the Berry phase from oscillatory peak positions in Fig. 1c. The intercept in the Landau fan diagram is found to be around $-1 / 8$, highlighting a nontrivial band topology of the $\alpha$ orbit $^{24,25}$.
To confirm that the main frequency $\alpha$ is stemming from the quasi-two-dimensional electronic pocket from $\mathrm{Mn}$ kagome lattice, we performed magneto-Seebeck measurements when the magnetic field is tilted away from the $c$ axis. As shown in Fig. 1g, both the oscillatory frequency $F$ and the cyclotron mass $m^{*}$ change in a $1 / \cos \theta$ manner with respect to the tilted angle $\theta$. For a gapped Dirac dispersion satisfying $E_{D}=\sqrt{\left(\hbar \nu_{D} k_{F}\right)^{2}+(\Delta / 2)^{2}}$, the oscillatory frequency and cyclotron mass should follow the relations ${ }^{26}$,

$$
F=\frac{E_{D}^{2}-(\Delta / 2)^{2}}{2 e \hbar \nu_{D}^{2} \cos \theta}, \quad m^{*}=\frac{E_{D}}{\nu_{D}^{2} \cos \theta}
$$

where $\Delta$ is the gap size, $v_{D}$ is the Fermi velocity of the Dirac cone. Because $\mathrm{TbMn}_{6} \mathrm{Sn}_{6}$ is a hard magnet with the easy axis pinned to the $c$ axis at low temperatures ${ }^{27-30}$, its $E_{D}, \Delta$ and $v_{D}$ change little when the external magnetic field is tilted away from the $c$ direction. The ratio $\mathrm{F} / \mathrm{m}^{*}$ is always close to $E_{D} / 2 \mathrm{eh}$, strongly supporting that the main quantum oscillation orbit $\alpha$ in transport is stemming from the Chern-gapped Dirac fermion. This is unlike the case of $\mathrm{Fe}_{3} \mathrm{Sn}_{2}$ whose electron mass and gap size can be easily tuned by an external magnetic field ${ }^{15,26,31}$. The strong coupling between $4 \mathrm{f}$ and $3 \mathrm{~d}$ electrons in $\mathrm{TbMn}_{6} \mathrm{Sn}_{6}$, which is absent in other transition-metal-bearing kagome magnets, guarantees a stable out-of-plane ferromagnetic $\mathrm{Mn}$ sublattice even in an extremely large external field and temperature range ${ }^{30}$. This large anisotropy makes the topological band of Chern-gapped Dirac fermion robust against the change of magnetic field and the elevated temperature, providing an ideal platform for studying Chern related topology.

Topological transverse transport. To further elucidate the quantum topology of this kagome magnet, we study the anomalous Hall effect, anomalous Nernst effect and anomalous thermal Hall effect. These three effects, stemming from the electron's anomalous velocity endowed by the Berry curvature field in magnets $^{3}$, are important fingerprints of the topological band structures residing near the Fermi energy. Figure $2 \mathrm{a}-\mathrm{c}$ shows the Hall conductivity $\sigma_{x y}$, Nernst thermopower $S_{x y}$ and thermal Hall conductivity $\kappa_{x y}$, which behave consistently with the $M(H)$ loops at various temperatures shown in Supplementary Fig. S1. Below $220 \mathrm{~K}$, sharp and large hysteresis loops can be observed in all three off-diagonal signals, corresponding to the spontaneous, outof-plane ferrimagnetic state. The hysteresis loops become narrow gradually and show additional bending as the in-plane magnetic components develop at higher temperature. Above around $310 \mathrm{~K}$, the three off-diagonal signals show the anomalous effects as long as the external field forces the in-plane moments to align along the $c$ axis ${ }^{27-29}$. The temperature dependence of the anomalous effects is summarized in Fig. 3d, e. An anomalous Nernst coefficient as large as $-2.4 \mu \mathrm{V} / \mathrm{K}$ is observed at $330 \mathrm{~K}$, that is comparable to the largest values reported in other topological magnets like $\mathrm{Co}_{2} \mathrm{MnGa}$ and $\mathrm{Co}_{3} \mathrm{Sn}_{2} \mathrm{~S}_{2}{ }^{32-35}$. The anomalous thermal Hall conductivity on the other hand, reaches $0.155 \mathrm{~W} / \mathrm{Km}$ at room temperature, much larger than those in topological magnets like $\mathrm{Mn}_{3} \mathrm{Sn}(0.04 \mathrm{~W} / \mathrm{Km})^{36}$ and $\mathrm{Mn}_{3} \mathrm{Ge}(0.015 \mathrm{~W} / \mathrm{Km})^{37}$ at $300 \mathrm{~K}$. These large anomalous transport terms at room temperature make $\mathrm{TbMn}_{6} \mathrm{Sn}_{6}$ a promising candidate for thermoelectric applications ${ }^{38-40}$.

In order to further study the intertwined relationships between these topological transport behaviors, we analyse their scaling relations. A conventional version in solids is the widely known Mott formula and the Wiedemann-Franz law, which associate the 

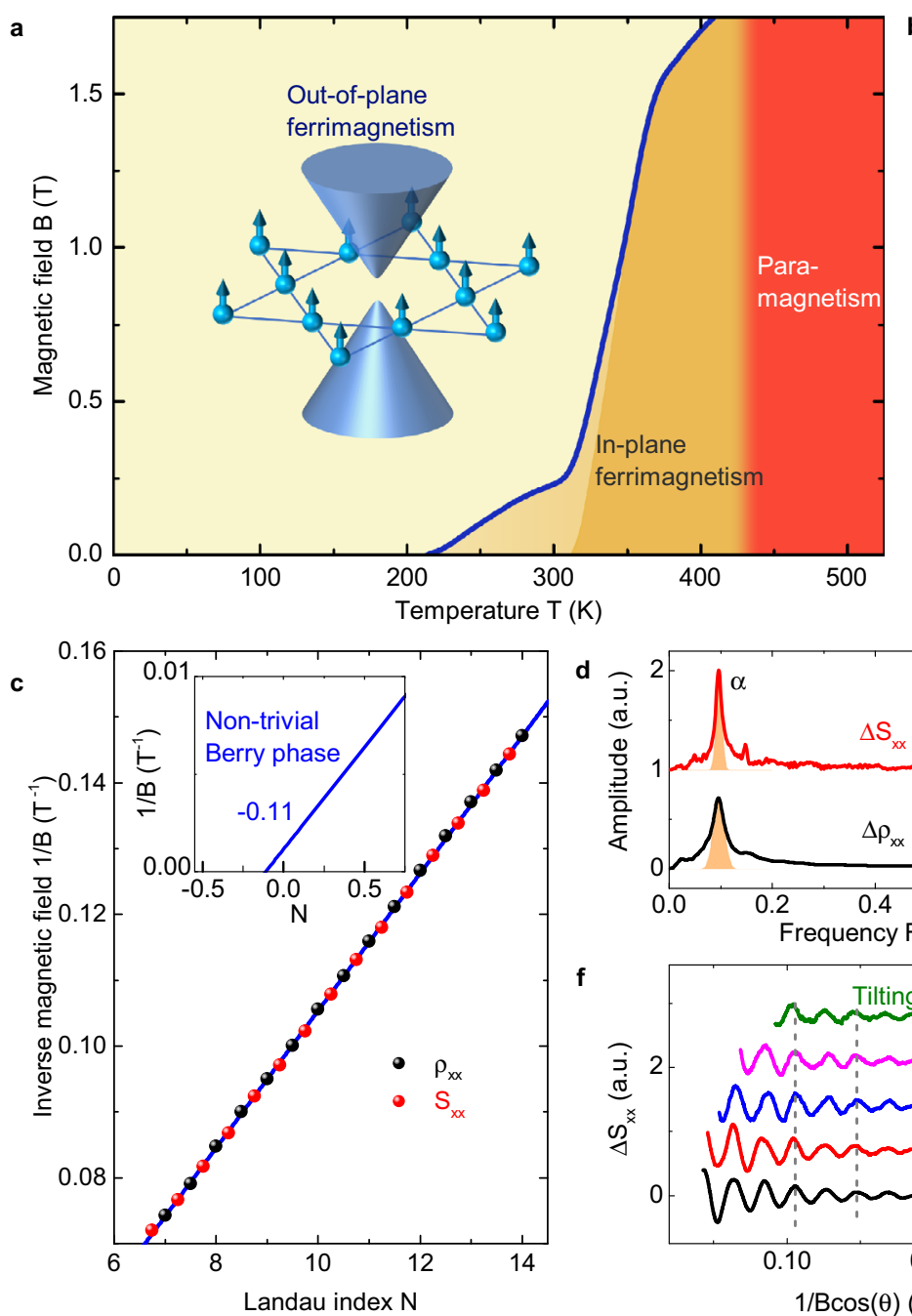

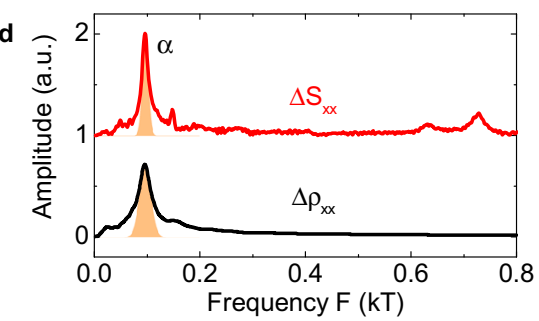

f

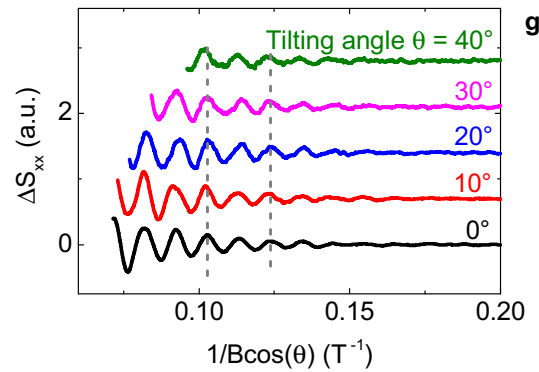

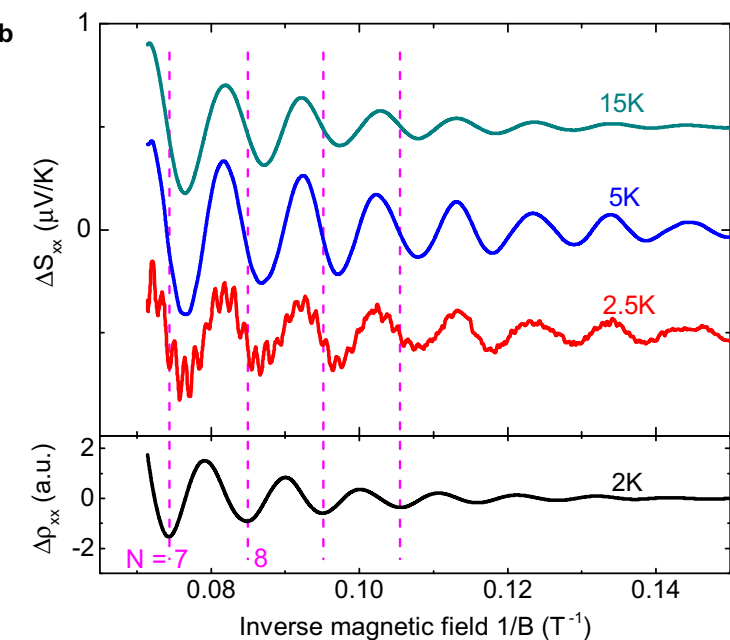
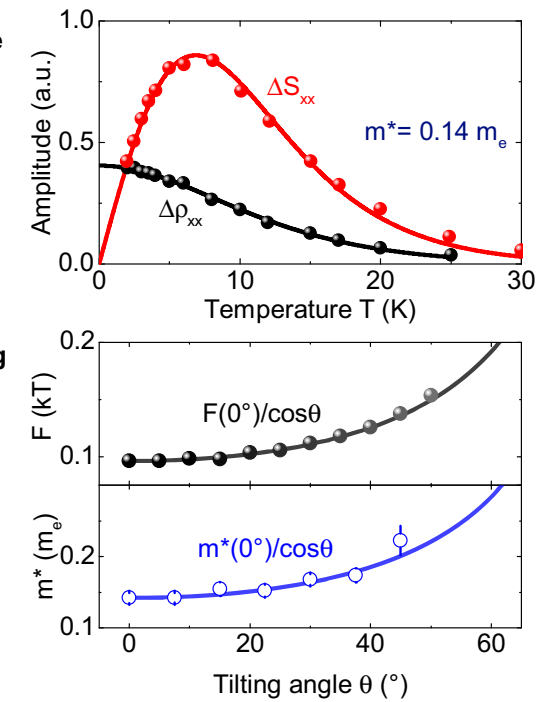

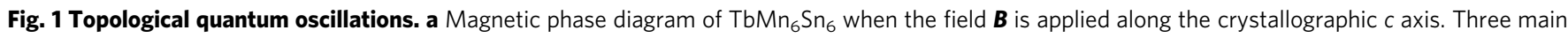
regions can be resolved, including the out-plane ferrimagnetic state where Chern-gapped Dirac states are supported, in-plane ferrimagnetic state, and paramagnetic state above $420 \mathrm{~K}$. Inset shows the magnetic Mn kagome lattice and corresponding Chern-gapped Dirac cone at the Brillouin Zone corner of the momentum space. b Quantum oscillations revealed in magneto-Seebeck signal $S_{x x}$ and magnetoresistance $\rho_{x x}$. There is a $\pi / 2$ phase shift between oscillatory parts of $S_{x x}$ and $\rho_{x x}$. c Landau fan diagram for the oscillations, suggesting a non-trivial Berry phase. d Fast Fourier transform spectrum of the oscillatory component in $S_{x x}$ and $\rho_{x x}$, showing dominant contribution of the $\alpha$ orbit. e Cyclotron mass fitting of oscillatory amplitude to the Lifshitz-Kosevich formulas. $\mathbf{f}$ Oscillatory parts in Seebeck signals when the field is tilted away from the $c$ direction. $\mathbf{g}$ Angle dependent oscillatory frequencies and corresponding cyclotron mass for orbit $\alpha$, both showing an inverse cosine behavior.

charge and entropy of the electron via ${ }^{1,2}$

$$
\begin{gathered}
\boldsymbol{\alpha}=\left.\frac{\pi^{2}}{3} \frac{k_{B}^{2} T}{e} \frac{\partial \boldsymbol{\sigma}}{\partial \varepsilon}\right|_{\varepsilon_{F}}, \\
\boldsymbol{\kappa}=\frac{\pi^{2}}{3} \frac{k_{B}^{2}}{e^{2}} \boldsymbol{\sigma} T=L_{0} \boldsymbol{\sigma} T,
\end{gathered}
$$

where $\varepsilon$ is the energy, $L_{0}=2.44 \times 10^{-8} \mathrm{~V}^{2} / \mathrm{K}^{2}$ is the Sommerfeld value, $\boldsymbol{\sigma}, \boldsymbol{\alpha}$ and $\boldsymbol{\kappa}$ are the electric, thermoelectric and thermal conductivity tensor, respectively. In the context of the anomalous transverse transport in topological magnets, however, their relevance remains much less explored.

As shown in Fig. 3a, we have confirmed that an intrinsic mechanism dominates in the anomalous Hall effect of $\mathrm{TbMn}_{6} \mathrm{Sn}_{6}$ by scaling $\sigma_{x y}^{A}$ versus $\sigma_{x x}$ in the presented temperature range ${ }^{16,17}$. A polynomial fitting ${ }^{41,42}$ as shown in the inset of Fig. 3a gives an intrinsic contribution of $\sigma_{x y}^{\text {int }} \sim 0.13 e^{2} / h$ per kagome layer. In accordance to the anomalous Hall conductivity and quantum oscillations of the $\alpha$ orbit, all parameters of the Chern-gapped Dirac fermion $\left(E_{D} \sim 130 \mathrm{meV}, \Delta=2 \sigma_{x y}^{i n t} E_{D} h / e^{2} \sim 34 \mathrm{meV}\right)$ can been experimentally nailed down and its Berry spectrum can be depicted. We can therefore trace the Berry curvature field to the Chern-gapped Dirac fermion in the stable out-of-plane ferrimagnetic state in $\mathrm{TbMn}_{6} \mathrm{Sn}_{6}$, which gives a unique opportunity to test the scaling relation of the $\sigma_{x y}^{A}, \alpha_{x y}^{A}$ and $\kappa_{x y}^{A}$ of the Chern-gapped Dirac fermion in a topological magnet.

The anomalous thermoelectric Hall conductivity is calculated as $\alpha_{x y}^{A}=\sigma_{x y}^{A} S_{x x}+\sigma_{x x} S_{x y}^{A}$. As shown in Fig. $3 \mathrm{c}$, the ratio $\alpha_{x y}^{A} / \sigma_{x y}^{A}$ in $\mathrm{TbMn}_{6} \mathrm{Sn}_{6}$ is small at low temperatures, and then shows an overall increase at elevated temperatures, reaching $-67 \mu \mathrm{V} / \mathrm{K}$ at $330 \mathrm{~K}$. For a Chern-gapped Dirac model as shown in Supplementary Notes 2, it can be treated analytically that $\partial \sigma_{x y}^{A} / \partial \varepsilon=\sigma_{x y}^{A} / E_{D}$. Substituting this into the Mott formula in 

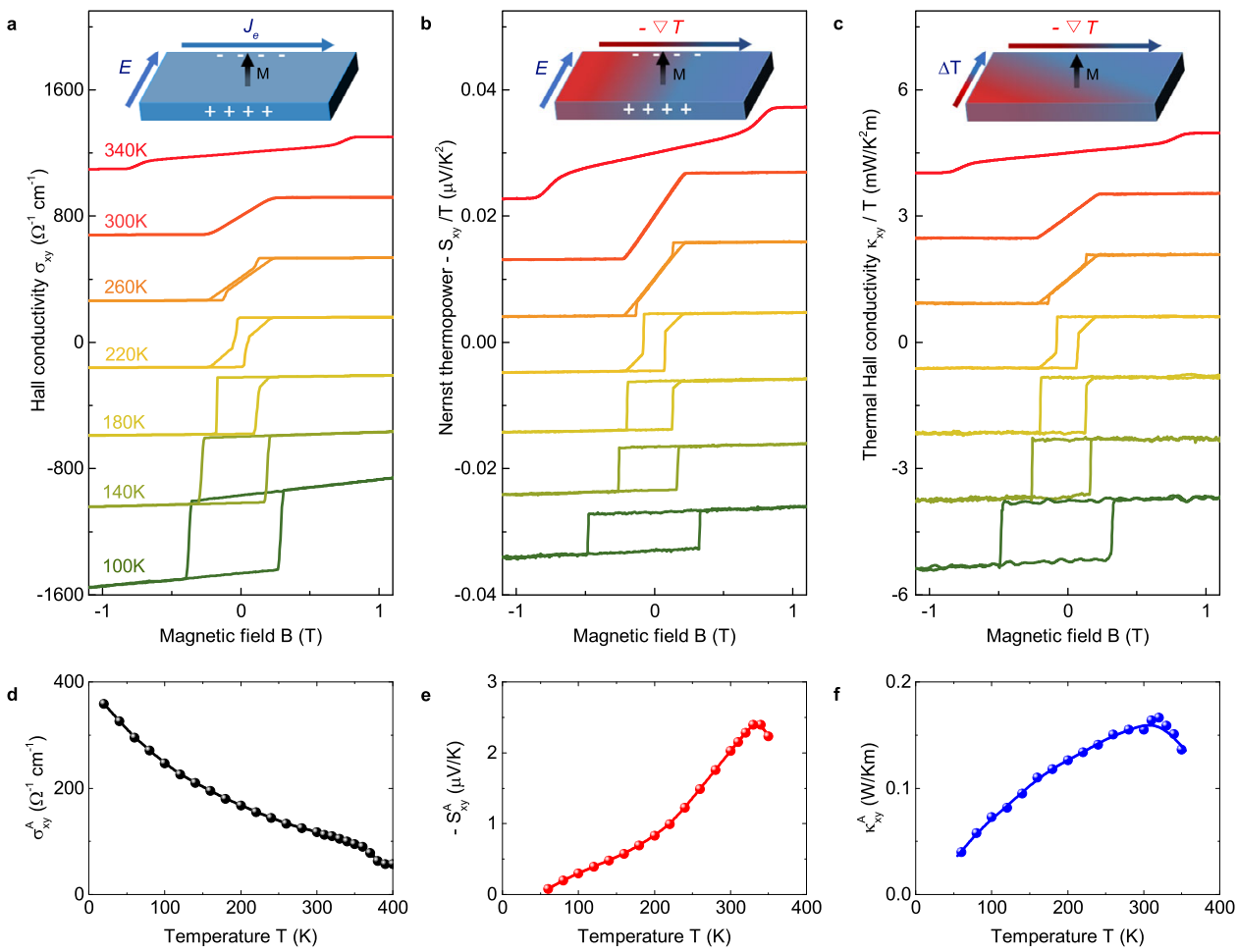

Fig. 2 Topological transverse transport. a-c The electric Hall conductivity $\sigma_{x y}$, the Nernst thermopower divided by temperature $-S_{x y} / T$ and thermal Hall conductivity divided by temperature $\kappa_{x y} / T$ at representative temperatures, showing dominant contribution of anomalous terms. Curves are shifted vertically for clarity. The resemblance of $\sigma_{x y}, S_{x y}$ and $\kappa_{x y}$ profiles indicates a shared origin from Berry curvature contributions. Insets show the sketches for anomalous Hall, Nernst and thermal Hall effect. d-f The temperature dependence of $\sigma_{x y}^{A}, S_{x y}^{A}$ and $\kappa_{x y}^{A}$, respectively.

Eq. (2), we have the relation between $\alpha_{x y}^{A}$ and $\sigma_{x y}^{A}$,

$$
\alpha_{x y}^{A} / \sigma_{x y}^{A}=\frac{\pi^{2}}{3} \frac{k_{B}}{e} \frac{k_{B} T}{E_{D}}
$$

The experimental data fit well into this simple linear line. The deviation around $100 \mathrm{~K}$ is currently unclear, and we speculate its origin from spin excitations ${ }^{43,44}$ as also evident in the magnetization susceptibility in Supplementary Fig. S1 which deserves future study. It has been suggested empirically that the ratio $\alpha_{x y}^{A} / \sigma_{x y}^{A}$ should be a sizable fraction of $\left|k_{B} / e\right| \sim 86 \mu \mathrm{V} / \mathrm{K}$ in topological magnets ${ }^{33,35}$. Our analysis shows that for the kagome Chern magnet, the physics behind the $k_{B} / e$ threshold is actually a competition between two energies: the thermal energy $k_{B} T$ and the Dirac cone energy $E_{D}$.

We now study the relation between the anomalous thermal Hall conductivity $\kappa_{x y}^{A}$ and anomalous Hall conductivity $\sigma_{x y}^{A}$. As shown in Fig. $3 d$, the ratio $\kappa_{x y}^{A} / \sigma_{x y}^{A}$ deviates from the linear $T$ dependence expected by the standard Wiedemann-Franz law. It apparently surpasses $L_{0} T$ above $100 \mathrm{~K}$, reaching 1.8 times $L_{0} T$ at $320 \mathrm{~K}$ and then the slope weakly damps at higher temperature. This substantial deviation can not be attributed to experimental errors, as the heat loss and geometric factors would only introduce at most $10 \%$ uncertainty in our study. It was reported that $\kappa_{x y}^{A} / \sigma_{x y}^{A} T$ remains close to $L_{0}$ in $\mathrm{Mn}_{3} \mathrm{Sn}$ and $\mathrm{Co}_{2} \mathrm{MnGa}$ when the Berry spectrum is smooth in the vicinity of the Fermi level ${ }^{33,36}$. In $\mathrm{Ni}$ and $\mathrm{Fe}$, the $\kappa_{x y}^{A} / \sigma_{x y}^{A} T$ deviates the Sommerfeld value downwards at higher temperatures due to the inelastic scattering 36,45 . A particular Berry spectrum also causes a suppressed $\kappa_{x y}^{A} / \sigma_{x y}^{A} T$ at high temperatures in $\mathrm{Mn}_{3} \mathrm{Ge}$ and $\mathrm{Co}_{3} \mathrm{Sn}_{2} \mathrm{~S}_{2} 37,46$. To the best of our knowledge, $\mathrm{TbMn}_{6} \mathrm{Sn}_{6}$ is the first topological magnet that possesses an enhanced $\kappa_{x y}^{A} / \sigma_{x y}^{A} T$ ratio at elevated temperatures. The inelastic scattering mechanism is self-evidently ruled out as it should cause an inverse effect. Considering the phonon Hall and magnon Hall effect is usually in the order of $10^{-3} \mathrm{~W} / \mathrm{Km}^{43,44,47,48}$, they are too small to account for this substantial enhancement. Below we show this anomaly in $\mathrm{TbMn}_{6} \mathrm{Sn}_{6}$ is due to the Berry curvature field effect hosted in the Chern-gapped Dirac fermions.

In transport theories, $\sigma_{x y}^{A}$ and $\kappa_{x y}^{A}$ can be expressed in the form of the Berry curvature $\Omega_{z}^{n}(\boldsymbol{k})^{49}$,

$$
\begin{gathered}
\sigma_{x y}^{A}=\frac{e^{2}}{\hbar} \int d \varepsilon\left(-\frac{\partial f}{\partial \varepsilon}\right) \tilde{\sigma}_{x y}(\varepsilon), \\
\kappa_{x y}^{A}=\frac{1}{\hbar T} \int d \varepsilon\left(-\varepsilon^{2} \frac{\partial f}{\partial \varepsilon}\right) \tilde{\sigma}_{x y}(\varepsilon), \\
\tilde{\sigma}_{x y}(\varepsilon)=\int_{B Z} \frac{d \boldsymbol{k}}{(2 \pi)^{3}} \sum_{\varepsilon_{n}<\varepsilon} \Omega_{z}^{n}(\boldsymbol{k}),
\end{gathered}
$$

where $f$ is the Fermi-Dirac distribution. In a Chern-gapped Dirac model, $\tilde{\sigma}_{x y}(\varepsilon)$ can be calculated as ${ }^{50}$

$$
\begin{aligned}
\tilde{\sigma}_{x y}(\varepsilon) & =\frac{1}{2 \pi} \frac{\Delta}{\sqrt{\Delta^{2}+4 \hbar^{2} k_{F}^{2} v_{D}^{2}}} \\
& = \begin{cases}\frac{1}{2 \pi} \frac{\Delta / 2}{\left|\varepsilon-E_{D}\right|}, & \left|\varepsilon-E_{D}\right|>\Delta / 2 \\
\frac{1}{2 \pi}, & \left|\varepsilon-E_{D}\right|<\Delta / 2 .\end{cases}
\end{aligned}
$$

As shown in Fig. $3 \mathrm{~b}$, the pondering functions in $\sigma_{x y}^{A}$ and $\kappa_{x y}^{A}$, namely $-\partial f / \partial \varepsilon$ and $-\varepsilon^{2} \partial f / \partial \varepsilon$ have different shapes. The former is a delta-like function, while the latter adopts a double-peak-like profile and is more extended in the energy scale. The Wiedemann-Franz law would only hold when $\tilde{\sigma}_{x y}(\varepsilon)$ is antisymmetric around zero energy. However in the case of a Cherngapped Dirac fermion, Berry curvature maximizes around the 

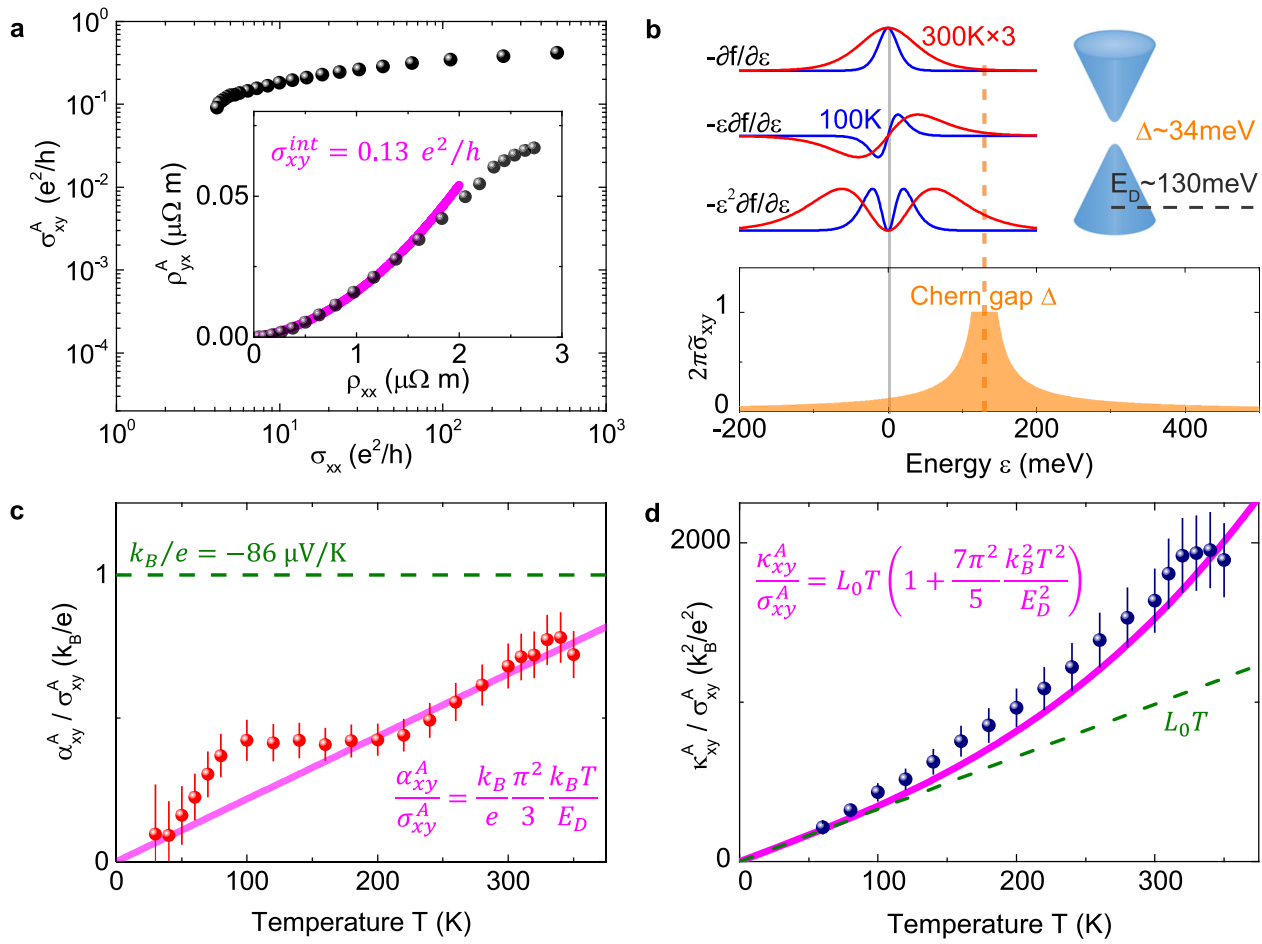

Fig. 3 Topological charge-entropy scaling. a Scaling of the anomalous Hall conductivity. The longitudinal conductivity $\sigma_{x x}$ for $\mathrm{TbMn}_{6} \mathrm{Sn}_{6}$ lies within the good metal region, suggesting a dominant intrinsic contribution. Inset shows a polynomial fitting of the intrinsic Hall conductivity, amounting to $0.13 e^{2} / h$ per kagome layer. b The pondering function for anomalous Hall conductivity $\sigma_{x y}^{A}$, anomalous thermoelectric Hall conductivity $\alpha_{x y}^{A}$, and the anomalous thermal Hall conductivity $\kappa_{x y}^{A}$ at $100 \mathrm{~K}$ and $300 \mathrm{~K}$, respectively, together with the $2 \pi \tilde{\sigma}_{x y}(\varepsilon)$ for the Chern gapped Dirac fermion with a gap size of $\Delta$. For clarity, the pondering functions at $300 \mathrm{~K}$ are multiplied by 3 times. Top-right sketch shows the Chern-gapped Dirac cone with gap size $\Delta \sim 34$ meV and Dirac cone energy $E_{D} \sim 130 \mathrm{meV}$. c The ratio $\alpha_{x y}^{A} / \sigma_{x y}^{A}$ at different temperatures, approaching $k_{B} / e$ at around $330 \mathrm{~K}$. This ratio scales with the linear function of $k_{B} T / E_{D}$, which is obtained from the Chern-gapped Dirac model. The error bars reflect the uncertainty in determining the sample size and low temperature anomalous Nernst signals. $\mathbf{d}$ The ratio $\kappa_{x y}^{A} / \sigma_{x y}^{A}$ at different temperatures. Above $100 \mathrm{~K}$, this ratio significantly enhances over the $T$-linear function expected by the Wiedemann-Franz law at elevated temperatures, which matches the $L_{0} T\left(1+\eta k_{B}^{2} T^{2} / E_{D}^{2}\right)$ behavior for the Chern-gapped Dirac model. The error bars come from the uncertainty in the sample's geometric factor.

Dirac gap, and contributes more in the latter integration as $-\varepsilon^{2} \partial f / \partial \varepsilon$ lies more close to the gap with increasing temperature. We note that it is the singularity of Berry curvature from the Chern Dirac gap that results in the enhancement of $\kappa_{x y}^{A} / \sigma_{x y}^{A}$. Using the Sommerfeld expansion as shown in Supplementary Notes 2 , it can be deduced that

$$
\kappa_{x y}^{A} / \sigma_{x y}^{A}=L_{0} T\left[1+\eta\left(\frac{k_{B} T}{E_{D}}\right)^{2}\right], \eta=\frac{7 \pi^{2}}{5} .
$$

The experimental ratios of $\kappa_{x y}^{A} / \sigma_{x y}^{A}$ fit well into the curves from the simple model.

\section{Discussion}

Due to the complex of magnetism and thermoelectric/thermal transport in real magnetic materials, it has been difficult to relate the anomalous Nernst effect and anomalous thermal Hall effect directly with the topological electrons. A common strategy is to calculate the energy dependence of the Berry curvature from $a b$ initio and thoroughly scan over the energy to find a rough match with the anomalous transverse effects in experiment. With the lack of the topological electrons' detailed information, the validity of this strategy, to a large extent, depends on the accuracy of the $a b$ initio calculation which is known to be difficult for correlated magnetic materials. In this work we have, for the first time, established a direct link between the anomalous effects and a particular topological fermion in a kagome magnet $\mathrm{TbMn}_{6} \mathrm{Sn}_{6}$. Starting from the angle-dependent quantum oscillations, we depict the two-dimensional Dirac dispersion. Then by comparing the anomalous transverse electric, thermoelectric and thermal transports, we are able to connect the anomalous effects to the Berry spectrum of the Chern-gapped Dirac fermion. In particular, two ratios, the $\alpha_{x y}^{A} / \sigma_{x y}^{A}$ and $\kappa_{x y}^{A} / \sigma_{x y}^{A}$, give inherent scaling relations. The former scales with $k_{B} T / E_{D}$ over an extended temperature range, while the latter, proportional to $T\left(1+\eta k_{B}^{2} T^{2} / E_{D}^{2}\right)$, enhances over the linear $T$-dependence above $100 \mathrm{~K}$. Only two parameters, the Dirac cone energy $E_{D}$ and the gap size $\Delta$ both of which are determined from experiments, are involved in our discussion, and the simple Chern-gapped Dirac model is able to capture most of the (anomalous) transport behaviors. Our results, therefore, establish a new model topological magnet for the proof-ofprinciple elaboration of the topological charge-entropy scaling. In future, it would be interesting to engineer this material down to atomic layers to realize higher temperature quantum anomalous Hall effect and to explore the topological scaling at the quantum-limit.

\section{Methods}

Material growth and magnetization measurement. Single crystals of $\mathrm{TbMn}_{6} \mathrm{Sn}_{6}$ were grown via a standard tin flux method ${ }^{29}$. Magnetic measurements on

$\mathrm{TbMn}_{6} \mathrm{Sn}_{6}$ were performed in a Quantum Design magnetic property measurement system (MPMS-3). When the field was applied along the $c$ axis, a sharp transition could be observed in the $M(T)$ profile at around $313 \mathrm{~K}\left(T_{S R}\right)$ in Supplementary Fig. S1, corresponding to the spin-reorientation transition. A magnetic anomaly occurs at around $100 \mathrm{~K}$ as indicated by the hump in the temperature derivative of the susceptibility, which suggest possible spin excitations in the kagome lattices ${ }^{43,44}$ and is beyond our current scope of research. 
Transport measurements. Transport properties of $\mathrm{TbMn}_{6} \mathrm{Sn}_{6}$ crystals were characterized in a Quantum Design physical property measurement system (PPMS-9) and a 14 T Oxford Teslatron PT system. Longitudinal and Hall resistivities were measured using a standard four-probe method. An AC Resistance Bridge (Model 372, Lakeshore) was used to measure the small electric signals. We use the convention $E_{i}=\rho_{i j} j_{j}$, namely $\rho_{y x}$ being positive for hole carriers.

For thermoelectric measurements, all samples were finely polished in order to reduce heat radiation. A one-heater-three-thermometer setup was used to monitor both the longitudinal and transverse temperature gradient. Temperature readings were acquired via Cernox thermometers below $50 \mathrm{~K}$ and type-E thermocouples above $50 \mathrm{~K}$. Heat flow was applied within the $a b$ plane. Vacuum better than $1 \times 10^{-4} \mathrm{~Pa}$ was maintained during the measurement. Thermal conductivity data were collected simultaneously. Seebeck and Nernst voltages were amplified by EM A10 and then collected by nano-voltmeter Keithley 2182A. The sign convention of $E_{i}=S_{i j} \partial_{j} T$ is adopted. In order to reveal the oscillatory components in $\rho_{x x}$ and $S_{x x}$ in Fig. 1, a smooth background has been subtracted.

Angle-dependent Seebeck signals at low temperatures were measured in the same magnets in a homemade vacuum chamber which can be rotated with respect to the magnetic field. The temperature gradient $(-\nabla T)$ was applied within the crystallographic $a b$ plane and perpendicular to the $a$ axis. Field is titled perpendicular to $-\nabla T$. The angle between $c$ axis and field direction is defined as $\theta$.

Cyclotron mass fitting. The cyclotron masses $\left(m^{*}\right)$ of the carriers were fitted by a standard and a modified Lifshitz-Kosevich formula ${ }^{22,23}$,

$$
\begin{gathered}
R_{T}^{\rho} \propto \frac{X}{\sinh (X)}, \\
R_{T}^{S} \propto \frac{(\alpha X) \operatorname{coth}(\alpha X)-1}{\sinh (\alpha X)},
\end{gathered}
$$

where $R_{T}^{\rho}$ and $R_{T}^{S}$ are the temperature-dependent oscillatory amplitude of resistivity and thermopower, respectively, and $\alpha=2 \pi^{2} k_{B} / e \hbar, X=m^{*} T / B$.

Reporting summary. Further information on research design is available in the Nature Research Reporting Summary linked to this article.

\section{Data availability}

The data that support the findings of this study are available from the corresponding author upon reasonable request.

Received: 5 November 2021; Accepted: 26 January 2022; Published online: 07 March 2022

\section{References}

1. Mott, N. F. et al. The Theory of the Properties of Metals and Alloys. Courier Dover Publications (1958).

2. Wiedemann, G. \& Franz, R. Relative conductivity of solids. Ann. Phys. Chem. 89, 497-531 (1853).

3. Xiao, D., Chang, M.-C. \& Niu, Q. Berry phase effects on electronic properties. Rev. Mod. Phys. 82, 1959-2007 (2010).

4. Keimer, B. \& Moore, J. The physics of quantum materials. Nat. Phys. 13, 1045-1055 (2017).

5. Sachdev, S. Topological order, emergent gauge fields, and Fermi surface reconstruction. Rep. Prog. Phys. 82, 014001 (2018).

6. Syôzi, I. Statistics of Kagomé Lattice. Prog. Theor. Phys. 6, 306-308 (1951).

7. Yin, J.-X., Pan, S. H. \& Hasan, M. Z. Probing topological quantum matter with scanning tunnelling microscopy. Nat. Rev. Phys. 3, 249-263 (2021).

8. Jiang, Y.-X. et al. Unconventional chiral charge order in kagome superconductor $\mathrm{KV}_{3} \mathrm{Sb}_{5}$. Nat. Mater. 20, 1353-1357 (2021).

9. Nakatsuji, S., Kiyohara, N. \& Higo, T. Large anomalous Hall effect in a noncollinear antiferromagnet at room temperature. Nature 527, 212-215 (2015).

10. Zhang, S. S. et al. Many-body resonance in a correlated topological kagome antiferromagnet. Phys. Rev. Lett. 125, 046401 (2020).

11. Liu, E. et al. Giant anomalous Hall effect in a ferromagnetic kagome-lattice semimetal. Nat. Phys. 14, 1125-1131 (2018).

12. Yin, J.-X. et al. Negative flat band magnetism in a spin-orbit-coupled correlated kagome magnet. Nat. Phys. 15, 443-448 (2019).

13. Yin, J.-X. et al. Spin-orbit quantum impurity in a topological magnet. Nat. Commun. 11, 4415 (2020).

14. Kida, T. et al. The giant anomalous Hall effect in the ferromagnet $\mathrm{Fe}_{3} \mathrm{Sn}_{2}-\mathrm{a}$ frustrated kagome metal. J. Phys. Condens. Matter 23, 112205 (2011).

15. Yin, J.-X. et al. Giant and anisotropic many-body spin-orbit tunability in a strongly correlated kagome magnet. Nature 562, 91-95 (2018).
16. Yin, J.-X. et al. Quantum-limit Chern topological magnetism in $\mathrm{TbMn}_{6} \mathrm{Sn}_{6}$. Nature 583, 533-536 (2020).

17. $\mathrm{Ma}, \mathrm{W}$. et al. Rare earth engineering in $\mathrm{RMn}_{6} \mathrm{Sn}_{6}(\mathrm{R}=\mathrm{Gd}-\mathrm{Tm}, \mathrm{Lu})$ topological kagome magnets. Phys. Rev. Lett. 126, 246602 (2021).

18. Lu, H.-Z. 3D quantum Hall effect. Natl Sci. Rev. 6, 208-210 (2018).

19. Haldane, F. D. M. Model for a quantum Hall effect without Landau levels: Condensed-matter realization of the "parity anomaly". Phys. Rev. Lett. 61, 2015-2018 (1988).

20. Kane, C. L. \& Mele, E. J. Quantum spin Hall effect in graphene. Phys. Rev. Lett. 95, 226801 (2005)

21. Xu, G., Lian, B. \& Zhang, S.-C. Intrinsic quantum anomalous Hall effect in the kagome lattice $\mathrm{Cs}_{2} \mathrm{LiMn}_{3} \mathrm{~F}_{12}$. Phys. Rev. Lett. 115, 186802 (2015).

22. Fletcher, R. On the amplitude of the quantum oscillations in the thermopower of metals. J. Low. Temp. Phys. 43, 363-370 (1981).

23. Shoenberg, D. Magnetic Oscillations in Metals Cambridge university press (2009)

24. Wang, C. M., Lu, H.-Z. \& Shen, S.-Q. Anomalous phase shift of quantum oscillations in 3D topological semimetals. Phys. Rev. Lett. 117, 077201 (2016).

25. Murakawa, H. et al. Detection of Berryas phase in a bulk Rashba semiconductor. Science 342, 1490-1493 (2013).

26. Ye, L. et al. de Haas-van Alphen effect of correlated Dirac states in kagome metal $\mathrm{Fe}_{3} \mathrm{Sn}_{2}$. Nat. Commun. 10, 4870 (2019).

27. Venturini, G., Idrissi, B. E. \& Malaman, B. Magnetic properties of $\mathrm{RMn}_{6} \mathrm{Sn}_{6}(\mathrm{R}$ $=\mathrm{Sc}, \mathrm{Y}, \mathrm{Gd}-\mathrm{Tm}, \mathrm{Lu})$ compounds with $\mathrm{HfFe}_{6} \mathrm{Ge}_{6}$ type structure. J. Magn. Magn. Mater. 94, 35-42 (1991).

28. Malaman, B. et al. Magnetic properties of $\mathrm{RMn}_{6} \mathrm{Sn}_{6}(\mathrm{R}=\mathrm{Gd}-\mathrm{Er})$ compounds from neutron diffraction and Mössbauer measurements. J. Magn. Magn. Mater. 202, 519-534 (1999).

29. Clatterbuck, D. \& Gschneidner, K. Magnetic properties of $\mathrm{RMn}_{6} \mathrm{Sn}_{6}(\mathrm{R}=\mathrm{Tb}$ Ho, Er, Tm, Lu) single crystals. J. Magn. Magn. Mater. 207, 78-94 (1999).

30. Zhang, L. Unusual Magnetic Behavior of Some Rare-earth and Manganese Compounds. Universiteit van Amsterdam [Host] (2005).

31. $\mathrm{Li}, \mathrm{Y}$. et al. Magnetic-field control of topological electronic response near room temperature in correlated kagome magnets. Phys. Rev. Lett. 123, 196604 (2019)

32. Sakai, A. et al. Giant anomalous Nernst effect and quantum-critical scaling in a ferromagnetic semimetal. Nat. Phys. 14, 1119-1124 (2018).

33. $\mathrm{Xu}, \mathrm{L}$. et al. Anomalous transverse response of $\mathrm{Co}_{2} \mathrm{MnGa}$ and universality of the room-temperature $\alpha_{i j}^{A} / \sigma_{i j}^{A}$ ratio across topological magnets. Phys. Rev. B 101, 180404 (2020).

34. Yang, H. et al. Giant anomalous Nernst effect in the magnetic Weyl semimetal $\mathrm{Co}_{3} \mathrm{Sn}_{2} \mathrm{~S}_{2}$. Phys. Rev. Mater. 4, 024202 (2020).

35. Ding, L. et al. Intrinsic anomalous Nernst effect amplified by disorder in a half-metallic semimetal. Phys. Rev. X. 9, 041061 (2019).

36. Li, X. et al. Anomalous Nernst and Righi-Leduc effects in $\mathrm{Mn}_{3} \mathrm{Sn}$ : Berry curvature and entropy flow. Phys. Rev. Lett. 119, 056601 (2017).

37. $\mathrm{Xu}, \mathrm{L}$. et al. Finite-temperature violation of the anomalous transverse Wiedemann-Franz law. Sci. Adv. 6, eaaz3522 (2020).

38. Mizuguchi, M. \& Nakatsuji, S. Energy-harvesting materials based on the anomalous Nernst effect. Sci. Technol. Adv. Mat. 20, 262-275 (2019).

39. Sakai, A. et al. Iron-based binary ferromagnets for transverse thermoelectric conversion. Nature 581, 53-57 (2020).

40. Li, X., Zhu, Z. \& Behnia, K. A monomaterial Nernst thermopile with hermaphroditic legs. Adv. Mater. 33, 2100751 (2021).

41. Zeng, C. et al. Linear magnetization dependence of the intrinsic anomalous Hall effect. Phys. Rev. Lett. 96, 037204 (2006).

42. Tian, Y., Ye, L. \& Jin, X. Proper scaling of the anomalous Hall effect. Phys. Rev. Lett. 103, 087206 (2009).

43. Hirschberger, M. et al. Large thermal Hall conductivity of neutral spin excitations in a frustrated quantum magnet. Science 348, 106-109 (2015).

44. Hirschberger, M. et al. Thermal Hall effect of spin excitations in a kagome magnet. Phys. Rev. Lett. 115, 106603 (2015).

45. Onose, Y., Shiomi, Y. \& Tokura, Y. Lorenz number determination of the dissipationless nature of the anomalous Hall effect in itinerant ferromagnets. Phys. Rev. Lett. 100, 016601 (2008).

46. Ding, L. et al. Quantum oscillations, magnetic breakdown and thermal Hall effect in $\mathrm{Co}_{3} \mathrm{Sn}_{2} \mathrm{~S}_{2}$. J. Phys. D: Appl. Phys. 54, 454003 (2021).

47. Strohm, C., Rikken, G. L. J. A. \& Wyder, P. Phenomenological evidence for the phonon Hall effect. Phys. Rev. Lett. 95, 155901 (2005).

48. Onose, Y. et al. Observation of the magnon Hall effect. Science 329, 297-299 (2010).

49. Vafek, O., Melikyan, A. \& Tešanović, Z. Quasiparticle Hall transport of $d$ -wave superconductors in the vortex state. Phys. Rev. B 64, 224508 (2001)

50. Sinitsyn, N. A. et al. Anomalous Hall effect in a two-dimensional Dirac band: The link between the Kubo-Streda formula and the semiclassical Boltzmann equation approach. Phys. Rev. B 75, 045315 (2007).

\section{Acknowledgements}

We thank Xiaokang Li and Zengwei Zhu for crosschecking our thermal transport data. X.X. thanks Yujin Zhang for assisting numerical calculations. The work was supported by the 
National Key R \& D Program of China grant number YFA0305601, National Natural Science Foundation of China grant numbers U1832214, 12141002, U2032213 and 12104461, and Strategic Priority Research Program of Chinese Academy of Sciences grant number XDB28000000. A portion of this work was supported by the High Magnetic Field Laboratory of Anhui Province. X.X. acknowledges support from the China Postdoctoral Science Foundation grant number 2020M682056, Anhui Postdoctoral Foundation grant number 2020B472, Anhui Provincial Natural Science Foundation grant number 2108085QA23, the HFIPS Director's Fund grant number YZJJ2021QN28, and Special Research Assistant Program, Chinese Academy of Sciences. H.-Z.L. was supported by the National Natural Science Foundation of China (11925402), Guangdong province (2016ZT06D348, 2020KCXTD001), Shenzhen High-level Special Fund (G02206304, G02206404), and the Science, Technology and Innovation Commission of Shenzhen Municipality (ZDSYS20170303165926217, JCYJ20170412152620376, KYTDPT20181011104202253), and Center for Computational Science and Engineering of SUSTech. T.-R.C. was supported by the Young Scholar Fellowship Program under a MOST grant for the Columbus Program, MOST110- 2636-M-006016, the Higher Education Sprout Project, Ministry of Education to the Headquarters of University Advancement at the National Cheng Kung University (NCKU), the National Center for Theoretical Sciences (Taiwan).

\section{Author contributions}

X.X. conducted the transport experiments in consultation with S.J., W.M., H.Z., and J.S. synthesized and characterized the samples; H.-R.T, P.V.S.R, T.-R.C. X.-B.Q, and H.-Z.L carried out the theoretical analysis in consultation with X.X., J.-X.Y., and S.J. X.X., J.-X.Y., Z.Q., and S.J. performed the data analysis and figure development and wrote the paper with contributions from all authors. Z.Q. and S.J. supervised the project. All authors discussed the results, interpretation, and conclusion.

\section{Competing interests}

The authors declare no competing interests.

\section{Additional information}

Supplementary information The online version contains supplementary material available at https://doi.org/10.1038/s41467-022-28796-6.

Correspondence and requests for materials should be addressed to Zhe Qu or Shuang Jia.

Peer review information Nature Communications thanks Yukako Fujishiro and the other anonymous reviewer(s) for their contribution to the peer review of this work.

Reprints and permission information is available at http://www.nature.com/reprints

Publisher's note Springer Nature remains neutral with regard to jurisdictional claims in published maps and institutional affiliations.

(c) (i) Open Access This article is licensed under a Creative Commons Attribution 4.0 International License, which permits use, sharing, adaptation, distribution and reproduction in any medium or format, as long as you give appropriate credit to the original author(s) and the source, provide a link to the Creative Commons license, and indicate if changes were made. The images or other third party material in this article are included in the article's Creative Commons license, unless indicated otherwise in a credit line to the material. If material is not included in the article's Creative Commons license and your intended use is not permitted by statutory regulation or exceeds the permitted use, you will need to obtain permission directly from the copyright holder. To view a copy of this license, visit http://creativecommons.org/ licenses/by/4.0/.

(C) The Author(s) 2022 